\title{
Die Rezeption der Werke Johann Georg Zimmermanns in Montpellier
}

Brigitte Lohff

\section{Summary}

At the end of the 18th century the French physicians discussed Johann Georg Zimmermann's medical concepts (i.e. medical experience, the influence of the soul on health and disease). In contrast to the German scientists, the French, especially those from the School of Montpellier, accepted Zimmermann's medical views as a confirmation of vitalism and neohippocratic medicine. In Germany, Zimmermann's medical works fell into oblivion after his death until the middle of 20th century. This may be a concequence of his intimate contacts to the European high nobility and of his polemic attacks against friends and enemies as well as his contempt for all forms of democracy and the French Revolution.

\section{Zusammenfassung}

Johann Georg Zimmermanns medizinische Werke (medizinischer Erfahrungsbegriff, Einfluss der seelischen Befindlichkeit auf Gesundheit und Krankheit) wurden von den französischen Ärzten, aber nicht bei den deutschen Ärzten intensiv diskutiert. Besonders die Vertreter der Schule von Montpellier sahen ihre Auffassung von Vitalismus und Neohippokratismus durch seine medizinischen Auffassungen bestätigt. Zimmermanns Werk geriet nach seinem Tod in Deutschland in Vergessenheit. Dies war mit eine Folge seiner engen Beziehung zu den Adelskreisen Europas, seiner polemischen Attacken gegen Freund und Feind und seiner vehementen Ablehnung demokratischer Ideen und der Französischen Revolution. Erst in den letzten

Prof. Dr. Brigitte Lohff, Abteilung Medizingeschichte, Ethik und Theoriebildung in der Medizin der MHH Hannover, Carl-Neuberg-Strasse 1, D-30625 Hannover 
Jahrzehnten findet eine erneute Auseinandersetzung mit seinen medizinischen Schriften statt.

«Zimmermann,Jean George:L'un des plus illustres médecins du dix huitième siècle» ${ }^{1}$, so wird der Hannoveraner Hof- und Leibmedicus Johann Georg Zimmermann (1728-1795) noch 30 Jahre nach seinem Tod in französischen Lexika charakterisiert. In Deutschland hatte Zimmermann nicht einen so hohen Ruf wie in Frankreich, wo er in eine Reihe mit dem «englischen Hippokrates» Thomas Sydenham, dem niederländischen Arzt Herman Boerhaave, dem Göttinger Professor für Anatomie, Botanik und Chirurgie Albrecht von Haller, dem französischen Arzt Théophile Bordeu und dem Professor der Medizin in Montpellier Jean Paul Barthez gestellt wurde.

Im Strom der Geschichte des 19. Jahrhunderts sind Zimmermanns medizinische Veröffentlichungen untergegangen. Seine Schrift «Von der Erfahrung in der Arzneykunst» wird kaum rezipiert ${ }^{2}$, und das vierbändige Werk «Über die Einsamkeit» findet auch nur sporadisch Erwähnung. Ausser von seinem Freund, Samuel A. A. D. Tissot, der unmittelbar nach seinem Tod 1797 die Biographie «La vie de Monsieur Zimmermann» verfasste ${ }^{3}$, lagen bis Ende des 19. Jahrhunderts zwei weitere umfassende Biographien vor: eine aus dem Jahr 1878 - zu seinem 150. Geburtstag - und eine historisch-kritische aus dem Jahr $1893^{4}$. Seine Briefwechsel mit dem europäischen Adel fanden Aufmerksamkeit und wurden zusammengetragen und veröffentlicht.

In den deutschen biographischen Lexika ${ }^{5}$ des 20. Jahrhunderts wird Zimmermann vornehmlich als popularphilosophischer Schriftsteller eingeordnet. In den letzten Jahrzehnten wird Zimmermanns medizinisches Werk

1 Dictionaire des sciences médicales. Biographie médicale.Tome 7, Paris 1825, S. 527-530.

2 Der dänische Arzt Julius Petersen geht in seinem Buch «Hauptmomente in der geschichtlichen Entwicklung der medicinischen Therapie» ausführlicher auf Zimmermanns medizinischen Erfahrungsbegriff ein. Petersen rückt Zimmermanns Auffassung in die Nähe des Mystikers Ringseis. Vgl. J. Petersen: Hauptmomente in der geschichtlichen Entwicklung der medicinischen Therapie. Hildesheim 1966, S. 133-137 (Reprint der Ausgabe Kopenhagen 1877).

3 Deutsche Übersetzung: «Das Leben des Ritters von Zimmermann, Hofrath und Leibarzt in Hannover», Hannover 1797.

4 Albrecht Rengger: Johann Georg Zimmermanns Briefe an einige seiner Freunde in der Schweiz. Aarau 1830; Eduard Bodemann: Johann Georg Zimmermann. Sein Leben und bisher ungedruckte Briefe an denselben ... Hannover 1878; Rudolf Ischer: Johann Georg Zimmermann's Leben und Werk. Bern 1893. Eduard Bodemann: Der Briefwechsel Katharina II von Russland und Joh. Georg Zimmermann. Hannover 1906.

5 In der Brockhaus-Encyklopädie wird er zudem als jemand bezeichnet, der sich polemisch gegen die Aufklärung und die katholische Weltanschauung wandte. Diese Kategorisierung geht sicher auf R. Ischers Beitrag in der Allgemeinen Deutschen Biographie zurück, wo Zimmermann als «Arzt und Popularphilosoph» vorgestellt wird (ADB, Bd. 45, 1900, S. 273-277). 
wieder ausführlicher diskutiert ${ }^{6}$. Mit seiner Dissertation «De irritabilitate» (1751) setzte sich Schmallenbach 1967 auseinander 7 . Anlässlich des 200. Todesjahres wurde an der Forschungs- und Studienstätte für Europäische Kulturgeschichte in Wolfenbüttel ein Arbeitsgespräch «Johann Georg Zimmermann, 1728-1795» organisiert $^{8}$. Zudem wurde Zimmermanns Leben und sein Werk in zwei Hannoveraner Ausstellungen in Erinnerung gebracht ${ }^{9}$. In einem umfangreichen Lesebuch sind Auszüge aus Zimmermanns Schriften heutigen Lesern wieder zugängig ${ }^{10}$.

Bei der geringen Beachtung, die Zimmermanns Werke in Kreisen der deutschen Mediziner nach seinem Tod fanden, ist es höchst erstaunlich, dass der Hof- und Leibmedicus des englisch-hannöverschen Herrscherhauses grössere Beachtung und Wertschätzung in Frankreich gefunden hat. Um diese Diskrepanz besser verstehen zu können, sei hier kurz auf sein Leben und seine Person eingegangen.

Johann Georg Zimmermann ${ }^{11}$, der am 8. Dezember 1728 in Brugg im Kanton Bern geboren wurde, hat 27 Jahre seines 67jährigen Lebens in Hannover gelebt. Medizin studierte er in Göttingen und wurde Schüler von Albrecht von Haller. Haller setzte sich für ihn menschlich und beruflich ein, was Zimmermann aus Hallers Sicht letztlich nicht zu würdigen wusste ${ }^{12}$. Nach der Promotion ging Zimmermann 1752 für vierzehn Jahre wieder nach Brugg und eröffnete dort eine Praxis. 1768 übernahm er nach Paul Gottlieb Werlhofs

6 Siehe z.B. Richard Toellner: Johann Georg Zimmermann (1728-1795). Der Arzt als Genie. Berichte zur Wissenschaftsgeschichte 2 (1979), S. 13-24; Erwin Ackerknecht: Johann Georg Zimmermann (1728-1795). Gesnerus 35 (1978), S. 224-229; Udo Benzenhöfer, Gisela vom Bruch (Hrsg.): Johann Georg Zimmermann: Von der Diät für die Seele. Hannover 1996.

7 Hans-Joachim Schmallenbach: Johann Georg Zimmermann und die Irritabilitätslehre. Diss med. Münster 1967.

8 Eine Veröffentlichung der Vorträge ist in der Reihe «Wolfenbüttler Forschungen» vorgesehen. Sie soll noch 1997 erscheinen.

9 «Seelenarzt und armer Tropf» Stadtarchiv Hannover (Oktober 1995; Fachbereich Informations- und Kommunikationswesen FH Hannover); «Johann Georg Zimmermann (1724-1795) und die Medizin des 18. Jahrhunderts» (Januar-April 1996; Abteilung Medizingeschichte, Ethik und Theoriebildung der Medizinischen Hochschule Hannover).

10 Andreas Langenbacher: Mit Skalpell und Federkiel - ein Lesebuch. Bern 1995.

11 Der kurze Abriss von Zimmermanns Leben basiert auf der in Anm. 4 aufgeführten Literatur.

12 Zimmermann wollte mit seinem Buch «Das Leben des Herrn von Haller», Zürich 1755 seinem Lehrer ein Denkmal setzen. Haller war mit dieser Veröffentlichung nicht ganz einverstanden, zumal sich späterhin auch das Verhältnis zwischen Lehrer und Schüler verschlechterte. Besonders Zimmermanns Begeisterung für Jean Jacques Rousseau wurde nicht von Haller geteilt. Vgl. dazu auch: Ischer, a.a.O. (Anm. 4), S. 224-225 und J. G. Zimmermanns Briefe an Haller, hrsg. von Rudolf Ischer [1751-1775]. Neues Berner Taschenbuch. Bern 1903-1911; Eduard Bodemann: Von und über Albrecht von Haller. Ungedruckte Briefe und Gedichte Hallers. Hannover 1885. 
$\operatorname{Tod}^{13}$ dessen Hof- und Leibmedicus-Stelle in Hannover, die er bis zu seinem Tod am 7. Oktober 1795 wahrnahm.

Zimmermann war zu seiner Zeit ein angesehener Arzt und genoss eine hohe Reputation in den Adelskreisen. Ebenso korrespondierte er mit vielen bedeutsamen Persönlichkeiten aus Literatur, Wissenschaft und Politik des 18. Jahrhunderts ${ }^{15}$. Zimmermann war eine Person, die Anstoss gab und erregte, und er war ein Mensch, der auf der Schattenlinie zwischen Anerkennung und Verzweiflung, Geselligkeit und tiefer Einsamkeit, wacher Weltzugewandtheit und Depression wanderte. Seine familiären Schicksalsschläge hätten selbst für ein schlichteres Gemüt Anlass zur Verzweiflung gegeben. Seine erste, von ihm sehr geliebte Frau starb 1770 nach 16 Ehejahren. Sein Sohn verfiel dem Wahnsinn, seine Tochter starb mit 25 Jahren an Tuberkulose.

Goethe und Zimmermann haben sich 1775 kennengelernt, aber nicht miteinander korrespondiert. In «Dichtung und Wahrheit» beschrieb er Zimmermann als von Natur aus heftig und geradeaus vor sich hin, als ungebändigten Charakter, von dem er in kurzer Zeit sehr viel gelernt habe. Und er ${ }^{16}$ fährt fort: «Ja dieser brave Mann führte bei äusserem Ansehen, Ruhm, Ehre, Rang und Vermögen das traurigste Leben und wer sich davon aus vorhandenen Druckschriften noch weiter unterrichten will, der wird ihn nicht verdammen, sondern bedauern. ${ }^{17}$ Am treffendsten und einfachsten hat diese widersprüchliche, komplizierte Person Johann Georg Zimmermann wohl seine zweite Frau in einem Brief an Tissot beschrieben: «Was würde das für ein Mann gewesen sein, wenn seine Nerven ihn niemals beherrscht

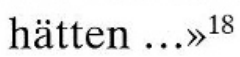

Weit über 100 Veröffentlichungen sind von Zimmermann verfasst worden. Neben den erwähnten Büchern und zwei Schriften über Friedrich den Grossen ${ }^{19}$ brachte ihm seine erste Buchveröffentlichung «Vom Nationalstolze»,

13 Udo Benzenhöfer: Der hannoversche Hof- und Leibarzt Paul Gottlieb Werlhof. Aachen 1992.

14 Goethe, Wieland, Lichtenberg, Moses Mendelssohn, Tissot, der in englischen Diensten stehende Naturforscher deLuc, Georg Forster, Albrecht von Haller, Lavater, Herder beschäftigten sich in literarischen Aperçus, Kommentaren, Kritiken und Briefen mit Zimmermanns Person, Veröffentlichungen, Äusserungen und Verhalten.

15 Vgl. dazu auch: Arthur Kielholz: Johann Georg Zimmermann zum zweihundertsten Geburtstag. Ein pathographischer Versuch. Imago 25 (1929), S. 241-262.

16 Zimmermann charakterisierte Goethe in einem Brief von 20. Nov. 1777 an Lavater: «Die Liebkosungen Goethes sind wie die eines Tigers. Man fasst unter seinen Umarmungen immer an den Dolch in der Tasche.» Bodemann a.a.O. (Anm. 4), S. 97.

17 Bodemann a.a.O. (Anm. 4), S. 95.

18 Bodemann a.a.O. (Anm. 4), S. 159.

19 Über Friedrich den Grossen und seine Unterredung mit ihm kurz vor seinem Tode. Leipzig 1788; Fragmente über Friedrich den Grossen, zur Geschichte seines Lebens, seiner Regierung und seines Charakters. Leipzig 1790. 
die sowohl ins Englische als auch ins Französische übersetzt wurde, damals viel Anerkennung. Zimmermann hat selber Schriften seines Freundes Tissot aus dem Französischen ins Deutsche übersetzt, da er Französisch wie eine zweite Muttersprache beherrschte. Ebenfalls hat er sich - wenn auch mit mässigem Erfolg - als Poet versucht und war bemüht, darin seinem Lehrer Haller zu gleichen.

Das «Hannöversche Magazin», ein 1750 gegründetes Intelligenz-Correspondenzblatt, war für Zimmermann ein wichtiges Journal, in dem er einen Teil seiner Schriften, Kritiken und Schmähschriften veröffentlichte. Über dieses Magazin und den «Göttinger Gelehrten Anzeiger» wurde auch die polemische Fehde zwischen Zimmermann und Lichtenberg über Lavaters Physiognomik ausgetragen. Der scharfzüngige Lichtenberg fühlte sich durch Zimmermanns vehementen Einsatz für Lavaters Physiognomie und die Ablehnung seiner - Lichtenbergs - lediglich kritischen Anmerkungen so angegriffen, dass er sich in unzähligen Aperçus höchst bissig über Zimmermann äusserte ${ }^{20}$.

Die Beziehungen Zimmermanns zum europäischen Adel gaben Anlass zu Bewunderung und zu spöttischen Bemerkungen. Andererseits wurden seine Beziehungen aber auch genutzt ${ }^{21}$. Zimmermann machte sich viele Feinde durch seine politischen Äusserungen. Für ihn, der republikanischen Ursprungs war, waren «Demokraten» eine Partei der Tollen. Alles, was mit der Revolution zusammenhing, war für ihn zutiefst verabscheuungswürdig und konnte nur Zerrüttung der bestehenden Ordnung bedeuten ${ }^{22}$.

Die Erfahrung, dass die eigenen Ansichten sich selber überleben, blieb Zimmermann nicht erspart, und er kämpfte verbissen und heftig bis zur Lächerlichkeit. Die Weltanschauung der Romantik und des Idealismus wur-

20 Vgl. Georg Christoph Lichtenberg, Sudelbücher Bd.1, Heft F. In: Ders. Schriften und Briefe hrsg. von Wolfgang Promies. München 1968.

21 Georg Forster wandte sich 1787 auf Anraten seines Schwiegervaters und Göttinger Professors für Klassische Philologie, Christian Gottlob Heyne, an Zimmermann. Da Zimmermann mit Katharina der Grossen in engem Briefkontakt stand, bat Forster, ihm behilflich zu sein. Der russische Hof hatte Forster angeworben, um eine Südsee-Expedition mit vorzubereiten, und ihn deshalb aus den polnischen Diensten befreit. Bedingt durch politische Umstände, wurde vergessen, ihm die versprochenen finanziellen Zuwendungen auszuzahlen. Zimmermann verwandte sich bei der Zarin für Forster und hatte Erfolg. Forster erhielt aber zur gleichen Zeit ein Angebot aus Mainz für eine Stelle als Kurfürstlicher Bibliothekar. Er nahm an und begeisterte sich für die Ideale der Französischen Revolution und galt damit als Vaterlandsverräter.

22 In der vollen Überzeugung der Richtigkeit seiner Ansicht veröffentlichte Zimmermann seine Ansichten in der Schrift - oder genauer in dem Pamphlet - «Mémoire an Seine Kaiserlichkönigliche Majestät Leopold den Zweiten über den Wahnwitz des Zeitalters und die Mordbrenner, welche Deutschland und Europa aufklären wollen» als Gabe zur Kaiserkrönung Leopold II., 1791.1995 gab Chr. Weiss diese Schrift in der Reihe «Kleines Archiv des achtzehnten Jahrhunderts» als Bd. 24 heraus. 
den die neuen Lebensphilosophien ${ }^{23}$. Zimmermanns schwieriger Charakter, seine Anerkennung in den Adelskreisen und die von ihm verfassten polemischen Schriften trugen mit dazu bei, dass seine medizinischen Schriften bei den deutschen Ärzten weniger Beachtung fanden. Um so bemerkenswerter ist es, dass im ausgehenden 18. Jahrhundert und bis ins 19. Jahrhundert hinein seine medizinischen Schriften und die darin vertretenen Auffassungen während der Zeit der Französischen Revolution in Frankreich geschätzt wurden.

\section{Zimmermanns medizinische Schriften im Spiegel der Thèses médicales an der Faculté de Médecine à Montpellier}

«Cette matière a été si bien traitée par Zimmermann, dans son premier volume sur l'experience ...». Dieser Kommentar findet sich in der Dissertation über eine erkenntnisphilosophische Methode, um Krankheiten zu deuten und zu verstehen ${ }^{24}$. Verfasst wurde diese Arbeit im Jahr IX des in Frankreich geltenden Republikanischen Kalenders; nach unserer Zeitrechnung also im Jahr 1800 oder $1801^{25}$.

Aber nicht nur in dieser Dissertation wird «Jean Georges» Zimmermann erwähnt, sondern auch in einer Dissertation über die Leidenschaften aus dem Jahr 1799, wobei der Verfasser sich auf das Buch «Über die Einsamkeit» bezieht $^{26}$.

Sehr ausführlich geht ebenfalls Felix Petetin in seiner Doktorarbeit zum Thema der Sensibilität ${ }^{27}$ auf Zimmermanns Dissertation aus dem Jahr 1751 ein. Darin vergleicht er u.a. die von Albrecht von Haller (1707-1777) beschriebenen und experimentell nachgewiesenen Phänomene von Irritabilität

23 Vgl. Henri Brunschwig: Gesellschaft und Romantik in Preussen im 18. Jahrhundert. Die Krise des preussischen Staates am Ende des 18. Jahrhunderts und die Entstehung der romantischen Mentalität. Frankfurt 1976.

24 P. C. Celliez: Réflexions philosophiques sur la méthode à employer pour parvenir la connoissance de la maladie. Thèse méd. Montpellier an IX.

25 Die Republikanische Kalenderrechnung wurde am 23. November 1793 auf Beschluss der Nationalversammlung eingeführt. Das Jahr I der Republikanischen Kalenderrechung begann mit dem 22. September 1792 (Herbst-Zeitgleichen) und endete am 21. September des folgenden Jahres. Die Monatsnamen wurden den jeweiligen Hauptereignissen des Jahres angepasst, so z. B. Vendémaire = August/September als die Monate der Weinlese (vendanges) oder Thermidor $=19$. Juli bis 18. August als Monat der Hitze. Bis Ende 1805 galt diese Kalenderrechnung; ab dem 1. Januar 1806 wurde wieder der Gregorianische Kalender eingeführt.

26 Laurent Beriot de Baguères: Quelques idées sur les passions. 24. Thermidor an VIII = 12. August 1799. Thèse méd. Montpellier.

27 Quelques considérations physiologiques sur la sensibilité. 27. Thermidor an VIII = 17. August 1799. Thèse méd. Montpellier. 
und Sensibilität ${ }^{28}$ mit den Ergebnissen aus Zimmermanns Dissertation «De irritabilitate» ${ }^{29}$. Petetin, der eine für damalige Zeiten ungewöhnlich umfangreiche Dissertation von 51 Druckseiten vorgelegt hat, folgerte, dass Zimmermanns Ergebnisse bedeutsamer seien als die von Haller. Zimmermann käme mit seinen Untersuchungen nicht wie Haller zu dieser rigiden Trennung von Irritabilität und Sensibilität. Haller hatte die Irritabilität als ein organisches Phänomen definiert, das ausschliesslich den Muskeln zukomme, während die Sensibilität nur in den Nerven vorzufinden sei. Zimmermann hingegen habe auch in anderen Teilen des Körpers irritable und sensible Eigenschaften nachweisen können ${ }^{30}$.

Um diese positive Bewertung bereits der Dissertation von Zimmermann in dieser französischen Thèse médicale einordnen zu können, soll kurz die Entwicklung des Vitalismus skizziert werden, denn die Bewertung der Zimmermannschen Arbeit ist nur im Zusammenhang mit der damaligen Diskussion um das Phänomen der Sensibilität/Irritabilität als Grundprinzip vitaler Prozesse zu verstehen. Gleichzeitig bildet die Vitalismus-Diskussion auch den Hintergrund dafür, weshalb Zimmermanns medizinische und ärztliche Auffassung in der Schule von Montpellier eine so positive Resonanz gefunden hat.

Im ausgehenden 17. Jahrhundert wurde neben der rein iatrophysikalischen Deutung des Lebens - d.h., der Organismus ist ein nach physikalischen Gesetzen der Hydromechanik funktionierender Körper - auch eine iatrochemische Deutung - d.h. die körperlichen Funktionen sind einzig und allein aus chemischen Prinzipien herzuleiten (Paracelsus, van Helmont) - vertreten. Doch mit diesen Organismusvorstellungen liessen sich nur sehr begrenzt vitale Prozesse beschreiben. Erzeugung neuen Lebens, Entwicklung, Nah-

28 Albrecht von Haller: De partibus corporis humani sensilibus et irritabilibus. [1752], in: Commentarii Societatis Regiae Scientiarum Gottingensis, Bd. 2, Gottingae 1753, S. 114-158.

29 Zimmermanns Dissertation wurde bereits vier Jahre später ins Italienische übersetzt: Dissertatione de Signor Gian Georgi Zimmermann sull'irritabilità. In: Sull'insensitività e irritabilità di alcune parti degli animali. Rom 1755, S. 71-132. Eine französische oder englische Übersetzung liegt nicht vor. Vgl. dazu auch Ischer a.a.O. (Anm. 4), S. 9-23.

30 Auf die scheinbar widersprüchliche Einschätzung der Zimmermannschen Dissertation seitens Hallers geht Gerhard Rudolph in seinem Beitrag: Hallers Lehre von der Irritabilität und Sensibilität. In: Karl Eduard Rothschuh (Hrsg.): Von Boerhaave bis Berger. Die Entwicklung der kontinentalen Physiologie im 18. und 19. Jahrhundert. Stuttgart 1964, S. 14-34 ein. Zimmermanns Beteiligung und Anteil an den Experimenten zur Irritabilität/Sensibilität, die er in seiner Dissertation von 1751 detailliert niedergeschrieben hat, werden von Haller bei seiner Ansprache vor der Göttingischen Gesellschaft der Wissenschaften am 22. April 1751 ausführlich gewürdigt. In dem 7. Band der Disputationes anatomicarum (1751) werden weder Zimmermanns Arbeiten noch diejenigen von Hallers Schülern aus dem Jahre 1746 zu diesem Themenkreis erwähnt. 
rungsumwandlung, Beseelung und der gesamte Bereich der seelischen Äusserungen und des Bewusstseins entzogen sich einer schlüssigen Erklärung mittels dieser Konzepte.

Der in Halle lehrende Professor der Medizin Georg Ernst Stahl (1659-1734) versuchte, die aus dem cartesianischen Dualismus entstandene iatromechanische Physiologie mit einem Konzept einer alles regierenden Anima zu überwinden. Im Organismus sollten zwar - so Stahl - die physikalischen oder chemischen Gesetze ihre Geltung behalten, aber die Belebtheit und Einzigartigkeit der organischen Welt lassen sich nur mittels spezifischer körpereigener Gesetzmässigkeiten erfassen. Gerade die Beobachtung, dass organische Materie ausserhalb des lebendigen Körpers zersetzbar ist, aber innerhalb des Organismus die Gärungs- und Zersetzungsprozesse im Zaum gehalten werden, bedurfte für Stahl, den Begründer der Phlogistontheorie, einer Erklärung ${ }^{31}$.

Stahls diesbezüglich formuliertes Konzept des Animismus fand wesentlich mehr Aufmerksamkeit bei den französischen Aufklärern ${ }^{32}$, während in Deutschland Friedrich Hoffmanns (1660-1742) mechanistisches Organismuskonzept intensiver verfolgt wurde. Der französische Arzt François Boissier de la Croix Sauvages (1706-1767) und Théophile Bordeu (1722-1774) griffen Stahls Konzept auf. Bordeu entwickelte es dahingehend, dass er nicht eine alles regierende Anima als lebensbedingendes Prinzip einführte, sondern den einzelnen Organen ihre «organspezifische» Vitalität zuordnete ${ }^{33}$. Dieses in Frankreich besonders vertretene Konzept des Vitalismus wurde in der «Encyclopédie» von Diderot und d'Alembert durch Ärzte und Wissenschaftler von der Faculté de Médecine in Montpellier verbreitet, da ihnen die Möglichkeit gegeben wurde, die umfangreicheren Artikel zur Physiologie und über Krankheitskonzepte zu schreiben ${ }^{34}$.

31 Georg Ernst Stahl: De synergia naturae in medendo. Halle 1695; Theoria vera medica. Halle 1706; insbesondere eine Auseinandersetzung und Ablehnung von Leibniz' Philosophie der prästabilierten Harmonie und der Monadenlehre in: Negotium otiosum seu IKIAMAXIA adversus positiones aliquis fundamentis theoria vera medica a Viro quodam celeberrimo. Halle 1720. Vgl. dazu Paul Hoffmann: La controverse entre Leibniz et Stahl sur la nature de l'âme. Stud. Voltaire 18 $8^{\text {th }}$ Cent. 199 (1981), S. 237-249. Zu Stahls Leben und Werk: Bernward Joseph Gottlieb: Bedeutung und Auswirkungen des hallischen Professors und königlich preussischen Leibarzt Georg Ernst Stahl auf den Vitalismus des 18. Jahrhunderts, insbesondere auf die Schule von Montpellier. Nova Acta Leopold. NF 12 (1943), S. 425-503; Irene Strube: Georg Ernst Stahl (Biographien hervorragender Naturwissenschaftler, Techniker und Mediziner, 76). Leipzig 1982.

32 Albert Lémoine: Le vitalisme et l'animisme de Stahl. Paris 1864.

33 Vgl. dazu: Gerhard Rudolph:Sur quelques conceptions physiologiques de Théophile de Bordeu (1722-1776). Actes de congrès des sciences 1985. Montpellier 1985, S. 34-45.

34 Jerome Proust: L'université et l'encyclopédie. In:Louis Dulieu (Hrsg.) : La médecine à Montpellier du XII ${ }^{\mathrm{e}}$ au XX' ${ }^{\mathrm{e}}$ siècle. Paris 1990, S. 135-140. 
Albrecht von Hallers Nachweis der irritablen und sensiblen Reaktionsweise spezifischer organischer Strukturen wurde von den meisten französischen und auch deutschen ${ }^{35}$ Ärzten, Physiologen und Philosophen in der zweiten Hälfte des 18. Jahrhunderts sofort als eindeutiger Nachweis verstanden, dass es körpereigene Gesetzmässigkeiten gebe, die ausschliesslich den lebenden Organismen zukommen. Die Mediziner hofften, mit diesem Phänomen den Gesetzen des Lebens auf der Spur zu sein, um irgendwann einmal, vergleichbar dem Gravitationsgesetz für die allgemeine Natur, ein sowohl gleich umfassendes als auch einfaches Naturgesetz für die organische Natur zu entdecken.

Mit den von Haller experimentell entdeckten irritablen oder sensiblen Äusserungen des Körpers schien dem gesuchten principe vital, der vis vitalis, der organischen Kraft oder der Lebenskraft, ein materiell organisches Korrelat zugeordnet werden zu können. Problematisch schien sowohl den französischen als auch den deutschen Ärzten Hallers strikte Einteilung in Irritabilität $=$ Muskelgewebe, Sensibilität $=$ Nervengewebe. Stück für Stück wurden nun Beweise vorgelegt, dass Sehnen, Muskeln oder z. B. die pia oder dura mater auch sensible neben irritablen Reaktionen zeigen.

Hinter all diesen Überlegungen stand, dass es den damaligen Ärzten sinnvoll schien, nur ein oberstes «Lebens»-Prinzip anzunehmen, das den Unterschied zwischen belebter und unbelebter Materie primär bedingt und dem alle anderen Kräfte (Reproduktion, Sekretion, Nahrungsumwandlung und auch die physikalischen Kräfte etc.) untergeordnet sind. In diesem Zusammenhang schien es ihnen vernünftig, der Sensibilität den Vorzug zu geben. Sie vermuteten, dass dahinter verborgen und/oder mit diesem Phänomen verknüpft das seit der Antike gesuchte Grundprinzip des Lebens zu finden sei. Zudem sei die Sensibilität ein vornehmlich den Menschen zukommendes Charakteristikum und könne deshalb auch nur als die hierarchisch an höchster Stelle stehende Lebenserscheinung gelten. Ob die Sensibilität allerdings die gesuchte Lebenskraft sei, war noch unentschieden.

In der Schule von Montpellier wurde besonders durch Paul Joseph Barthez ${ }^{36}$ (1734-1806) der französische Vitalismus auf der Basis der Philosophie der Aufklärung (Bacon, Montaigne, Cabanis) und hippokratischer Ideen wei-

35 Vgl. dazu auch Brigitte Lohff: Die Suche nach der Wissenschaftlichkeit der Physiologie in der Zeit der Romantik. Ein Beitrag zur Erkenntnisphilosophie der Medizin (Medizin in Geschichte und Kultur, 17). Stuttgart 1990; dies.: The concept of vital force as a researchprogram. Episteme (im Druck); dies.: Did the concept of vital force hinder scientific progress between 1750 and 1830 ? J. Hist. Biol. (im Druck).

36 Paul Joseph Barthez, geb. 2. Dez. 1734 in Montpellier, studierte dort auch Medizin. Er wird nach seiner Approbation 1753 Militärarzt und gleichzeitig zum Königlichen Zensor ernannt. 
terentwickelt. Barthez fand in Montpellier eine grosse Anzahl von Anhängern, die seine Idee des Vitalismus in Forschung und Lehre vertraten. Dieser Vitalismus war nicht nur ein theoretisches Konzept zur Erklärung der organischen Funktionen, sondern es wurde auch zum Verstehen von Krankheitsentstehungen und -behandlungen herangezogen ${ }^{37}$. In der Schule von Montpellier wurde zudem ärztliches Denken und Handeln auf dem Hintergrund einer Rückbesinnung auf die hippokratischen Ideale gelehrt. Die medizinische Auffassung der Schule von Montpellier lässt sich wie folgt zusammenfassend charakterisieren:

1) Oberstes Gebot ist ärztliche Beobachtung und Erfahrung.

2) Es ist anzunehmen, dass ein principe vital das Leben gegenüber der toten Materie bestimmt.

3) Es ist die Fähigkeit des Organismus zu nutzen, mit natürlichen Mitteln die Selbstheilungskräfte (das principe vital) zu unterstützen.

In allen diesen Punkten trafen sich die Auffassungen der Schule von Montpellier mit denen von Zimmermann. In seinem Buch «Von der Erfahrung in der Arzneykunde» hatte er ausdrücklich die Bedeutung der ärztlichen Beobachtung hervorgehoben. So grenzt er z.B. Erfahrung und Experiment gegeneinander ab: «Ein Arzt, der den natürlichen Verlauf einer Krankheit betrachtet, macht also Beobachtungen, ein Arzt, der in einer Krankheit ein Mittel gibt und auf die Wirkungen dieses Mittels aufmerksam ist, macht ein Experiment. Der beobachtende Arzt hört die Natur, der erfahrende frägt sie» ${ }^{38}$. Das entsprach in vollem Umfang der von Barthez geforderten Rückbesinnung auf die ärztliche Beobachtung ${ }^{39}$. In diesem Sinne argumentierten auch die Medizinstudenten in ihren vorgelegten Thèses médicales. Ihre Lehrer an

Barthez studierte nebenbei Jura und promovierte in diesem Fach 1780. Ab 1759 unterrichtete er Physiologie, dann Therapie, Pathologie und auch Anatomie und Botanik an der Universität in Montpellier. 1778 übernahm er den Lehrstuhl für Anatomie und Botanik. Unter Napoleon wurde er zum Gouvernements-Arzt des Kaisers ernannt. Er starb am 15. Oktober 1806 in Paris. Seine Bücher «Nouvelle méchanique de l'homme et des animaux» 1795 und «Nouveaux éléments des sciences des hommes» 1806 sind Grundlage des Montpellierer Vitalismus in der spezifischen Ausprägung des Barthezianismus. Vgl. dazu auch: Thierry Lavabre-Bertrand: Barthez et le Vitalisme. In: Louis Dulieu (Hrsg.): La médecine à Montpellier du XII ${ }^{\mathrm{a}}$ au XX ${ }^{\mathrm{e}}$ siècle. Paris 1990, S. 142-144.

37 Brigitte Lohff: Self-healing forces and concepts of health and disease. A historical discourse. Theoretical medicine (im Druck).

38 Johann Georg Zimmermann: Von der Erfahrung in der Arzneykunde. Neue Aufl. Zürich 1787, S. 26. Vgl. dazu auch: Antoinette Emch-Dériaz: À propos de l'expérience en médecine de Zimmermann. Can. Bull. Med. Hist. 9 (1992), S. 3-15.

39 Paul Joseph Barthez: Nouveaux éléments des sciences des hommes. $2^{\mathrm{e}}$ Ed. Montpellier 1806; ders.: Discours sur le génie d'Hippocrate (4 Mess. an IX= 24. Juni 1801). In: Recueil de discours. Montpellier 1820, S. 59-108. 
der Faculté de Médecine Chaptal ${ }^{40}$, Dumas ${ }^{41}$ und Barthez, haben ihnen diese ärztliche Auffassung vermittelt. Sie verwiesen in diesem Zusammenhang auch auf den deutschen Arzt Zimmermann. Dieser habe stets kritisiert: «Les objects qu'il examine ne font réfléchir les traits de celui qui les observe.» ${ }^{42}$

Ebenso hoben sie in ihren Begründungen hervor, dass Zimmermann stets darauf hingewiesen habe, dass ein Arzt erst einmal die Krankheiten beobachten müsse, bevor er deren Ursachen bestimmen wolle. Letztlich müssten die Ärzte zu der Einsicht gelangen: «La doctrine des causes des maladies est la science philosophique de la médecine.» ${ }^{43}$

Aufgrund dieser von Zimmermann vertretenen Auffassung kann er in eine Reihe mit Hippokrates, Celsus und der Schule von Montpellier gestellt werden, wie Jean Jacques Lordat, späterer Lehrstuhlinhaber für Physiologie in Montpellier ${ }^{44}$, in seiner Dissertation von 1797 schreibt:

«Il constate par l'observation que chaque homme a un organe relativement affoibli, et vers lequel le Principe de Vie semble diriger un appareil des movemens, toutes les fois qu'il s'opère quelque changement extraordinaire

40 Jean Antoine Chaptal (1756-1830) studierte Medizin in Montpellier und war Befürworter der dort vertretenen Auffassung des Vitalismus. 1777 ging er für drei Jahre nach Paris und studierte dort Chemie. 1781 wurde der 25jährige Chaptal Professor der Chemie in Montpellier.Zwischen 1795-1800 war er Professor der Chemie an der neugegründeten École de Santé und an der École polytechnique. Von 1800-1804 wurde er Minister für Innere Angelegenheiten. Auf seine Anregung hin wurde in Frankreich ein Amt für Statistik eingeführt. Chaptal war Minister unter Ludwig XVI., Napoleon und Ludwig XVIII. Zu Chaptal vgl. Michel Pérounet: Un grand chimiste: Jean Antoine Chaptal. In: Louis Dulieu (Hrsg.): Médecine à Montpellier des XII ${ }^{\mathrm{e}}$ au XX ${ }^{\mathrm{e}}$ sciècle. Paris 1990, S. 186-188.

41 Charles-Louis Dumas (1765-1813) studierte Medizin in Montpellier. Nach Schliessung der Universität (1792) übernahm er 1795 den Lehrstuhl für Anatomie an der École de Santé in Montpellier und wurde 1807 deren Direktor. 1808 wurde die Universität Montpellier wieder eröffnet und Dumas Dekan der Faculté de Médecine.

42 Die richtige ärztliche Beobachtung, wie sie Zimmermann beschrieben hat, stellt ihn in eine Reihe mit den Erneuerern der Medizin de Haen, Cullen und Bordeu. Henri Pélip: Abus des méthodes exclusives en médecine et moyens de les éviter. 1. Thermidor an V=20. September 1797. Thèse méd. Montpellier, S. 32; 37.

43 Pélip a.a.O., S. 37.

44 Jacques Lordat (1773-1870) erhielt seine medizinisch-praktische Ausbildung bei Broca in Montpellier und wurde 1794 〈Chirurgien 3ème classe`. 1795 schrieb er sich als freier Zuhörer (Gaststudent) an der École de Santé ein. Nach Abschluss seiner Thèse médicale wurde er Sanitätsoffizier. Ab 1799 bot er private Kurse für Anatomie, Physiologie an. 1811 wurde er Nachfolger von André Méjan auf dem Lehrstuhl für operative Medizin; 1813 Nachfolger von Jean-Louis Dumas als ordentlicher Professor für Physiologie und Anatomie. Ab 1824 bis $\underline{1860}$ ! vertrat er den Lehrstuhl für Physiologie. Hoch geehrt, aber bereits vergessen, starb er am 25. April 1870. Lordat war der treueste Anhänger und Verteidiger Barthez', der Lordat alle seine Schriften nach dem Tod überlassen hatte. Zu Lordat vgl. Thierry Lavatre-Bertrand; André Bandin: Lordat et le vitalisme philosophique. In: Louis Dulieu (Hrsg.) Médecine à Montpellier des XII ${ }^{\mathrm{e}}$ au XX ${ }^{\mathrm{e}}$ sciècle. Paris 1990, S. 202-203. 
dans l'Economie. Cette vérité dont on trouve quelques fondemens dans Hippocrates, a été confirmée par Celse, et renouvellée par Zimmermann, Barthez et son école.» ${ }^{45}$

Zimmermanns Schrift «Über die Einsamkeit» und deren Auswirkungen auf das seelische und körperliche Befinden ${ }^{46}$ entsprach dem von Philipp Pinel beschriebenen Einfluss der Seele auf das allgemeine Befinden und die Entstehung bzw. Vermeidung von Geisteskrankheiten. Folgende Zielsetzung verfolgte Zimmermann dabei: «Die Anzeige zur Wegnahme der Ursachen der Krankheiten ist durch die Diät für den Leib noch nicht erfüllt. Wir müssen auch die Wege aufspüren, auf welche man die Ursachen der Krankheiten von der Seele wegnehmen oder selbst durch die Seele heben kann.» ${ }^{47}$ In dem Manuskript «Von der Diät der Seele», welches als dritter Band des Werkes «Von der Erfahrung der Arzneykunde» erscheinen sollte, vertrat Zimmermann ebenfalls wie auch in den gedruckten Bänden eine vorsichtige, an diätetischen Regeln orientierte Behandlungsmethode ${ }^{48}$. Dass es einen direkten Zusammenhang zwischen der Seele und dem Körper gebe und deshalb die Krankheiten wechselseitig verursacht werden können, ist nach Zimmermann die «demütigere Philosophie der Ärzte schlechterdings» ${ }^{49}$.

Neben der Dissertation von Zimmermann, seinem Buch über die Einsamkeit und über die Erfahrung findet auch seine Schrift «Von der Ruhr unter dem Volke» ausführlich Erwähnung, wie den Dissertationen aus Montpellier ab $1785 \mathrm{zu}$ entnehmen ist ${ }^{50}$. Hervorzuheben ist, dass seine wichtigen medizinischen Schriften wenige Jahre nach dem Erscheinen ins Französische übersetzt wurden, so dass die Studenten sich in ihrer Sprache mit den Gedanken dieses Arztes auseinandersetzen konnten ${ }^{51}$.

45 Jaques Lordat: Réflexions sur la nécessité de la physiologie dans l'étude et l'expérience. 18. Thermidor an $5=8$. August 1797, Thèse méd. Montpellier, S. 50 .

46 Soweit mir bekannt, gibt es nur eine einzige ausführliche Auseinandersetzung zu Zimmermanns Buch in Deutschland - Leo Maduschka: Das Problem der Einsamkeit im 18. Jahrhundert im besonderen bei J. G. Zimmermann. [Forschungen zur neueren Literaturgeschichte, 66] Weimar 1933.

47 Zit. nach: Benzenhöfer, vom Bruch, a.a.O. (Anm. 6), S. 39.

48 Die von Zimmermann vorgeschlagenen Behandlungsmethoden veranlassten Friedrich Casimir Medicus zu folgender Veröffentlichung: F. C. Medicus: Brief an den Herrn Johann Georg Zimmermann über einige Erfahrungen aus der Arznei-Wissenschaft. Mannheim 1766.

49 Von der Diät für die Seele, a.a.O. (Anm. 6), S. 40.

50 Amable Frayssinous: Mes doutes ou réflexions médicales suivies de quelques considérations sur la force cellulaire. An VII (1799/1800) Thèse méd. Montpellier, S. 40. P. C. Celliez: Réflexions philosophiques sur la méthode à employer pour parvenir à la connoissance de la maladie. An IX (1802/1803) Thèse méd. Montpellier, S. 22. Vgl. dazu auch Louis Dulieu: Index des auteurs cités dans les catalogues des thèses. In ders.: Médecine à Montpellier. Tome 3,2: L'époque classique. Montpellier 1966, S. 1139-1145.

51 Zitate aus Zimmermanns Schriften werden stets in Französisch und nicht in Deutsch in den Dissertationen wiedergegeben. 
Folgende Werke Zimmermanns konnten von den Studenten an der Faculté des Médecine Ende des 18. und im frühen 19. Jahrhundert gelesen werden:

1: Von der Erfahrung in der Arzneikunst. 2 Bde. Zürich 1763-1764

2: Traité de l'expérience en général, et en particulier dans l'art de guérir, traduit de l'allemand par Lefebvre de Villebrune. Paris: Vicente $1774^{52}$

3: Von der Ruhr unter dem Volke im Jahre 1765. Zürich $1767^{53}$

4: Traité de dysenterie épidémique, traduit de l'allemand par Lefebvre de Villebrune. Paris: Vicente $1775^{54}$

5: Traité de dysenterie. Nouvelle édition Lausanne: Libraires associés 1794

6: Vom Nationalstolze. Zürich $1758^{55}$

7: Méditation sur la solitude, 2 ed. traduit de allemand par A. J. L. Jourdan. Paris: Ballière $1825^{56}$

Dass Zimmermann in Frankreich geschätzt wurde, lässt sich, wie bereits erwähnt, auch anhand der biographischen Lexika ersehen. So kam Zimmermann bereits zu seinen Lebzeiten hohes Ansehen zu: «Zimmermann est un de ces hommes nés pour le bien de l'humanité, \& qui a essuyé, comme tant d'habiles gens, les traits malins des erreurs populaires: aussi démasque-t-il bien ces erreurs ...», und bezüglich des Buches über die Erfahrung wird geurteilt: «Les gens du monde y trouveront un excellent préservatif contre empirisme \& charlatanerie, ils y apprendront à distinguer la vraie Médecine. ${ }^{57}$

Aber auch noch nach Zimmermanns Tod wird er z.B. in dem Dictionaire des Sciences médicales von 1825 gewürdigt (s.o.). Ebenfalls ausführlich wird Zimmermann noch 1866 in der «Nouvelle Biographie générale depuis les temps les plus reculés jusqu'à nos jours ${ }^{58}$ » behandelt. Allerdings wird dort auf seine Werke über Friedrich den Grossen kritischer hingewiesen, da Zimmermanns politische Haltung in jener Zeit distanzierter betrachtet wurde.

52 Eine Neuübersetzung wurde von dem Montpellierer Bibliothekar Gabriel Prunelle 1818 vorgenommen.

53 Welche Bedeutung der Arbeit über die Ruhr in Frankreich eingeräumt wurde, lässt sich daran erkennen, dass sie in der dreissigbändigen «Bibliothèque classique médicale» erschienen ist: Traité de l'expérience et de la dysenterie. (Encyclopédie des sciences médicales, ou traité général méthodique et complêt des divers branches de l'art de guérir par M. M. Alibert, Barbier, Bayle etc.) Vol. 11, Paris 1852.

54 Zweite Auflage, 1788.

55 «De l'orgueil», Paris 1769.

56 Die erste Übersetzung ins Französische erfolgte 1790 durch J. P. Mercier.

57 Dictionaire historique de la Médecine ancienne et moderne par Nicolas François Joseph Eloy. Tome 4, Mons 1778, S. 61.

58 Nouvelle Biographie générale depuis les temps les plus reculés jusqu'à nos jours. Tome 4 , Paris: 1866 , S. 723-724. 
Dass aber die Studentengenerationen der ersten Französischen Republik kurz nach der Französischen Revolution diesbezüglich nicht seine extrem reaktionäre politische Haltung bewerten, sondern seine medizinisch-ärztliche Auffassung besonders schätzen, ist bemerkenswert. Ausschlaggebend dürfte die grosse Übereinstimmung zwischen dem ärztlichen Erfahrungsbegriff des Hannoveraner Leib- und Hofarztes mit dem von Barthez und der Schule von Montpellier sein. Dass Zimmermann den Ideen von Rousseau positiv gegenüberstand, wurde bereits erwähnt ${ }^{59}$. Die positive Auseinandersetzung mit seinen medizinischen Schriften an der Schule von Montpellier und die daraus abgeleitete Bestätigung des Konzepts des französischen Vitalismus lässt sich aber auch daraus ableiten, dass Zimmermann sich gerade in dem Buch «Von der Erfahrung» auf Bacon, Locke, Hippokrates und Sydenham berief ${ }^{60}$. Diese Autoren waren für Barthez und seine Schüler die zentralen Bezugspunkte für das ärztliche Verhalten und Medizinverständnis.

Bei den deutschen Ärzten wurde die Person Zimmermann und in Folge dessen auch sein Werk aufgrund seiner rigorosen Ablehnung der demokratischen Bewegungen und der revolutionären Veränderungen am Ende des Jahrhunderts, seiner engen Verflechtung mit dem europäischen Adel und seines höchst schwierigen Charakters mit Missachtung gestraft, so dass seine Schriften und sein ärztliches Denken nach seinem Tod kaum noch Beachtung fanden. Diese Ächtung empfanden bereits einige Zeitgenossen Zimmermanns als zu weitgehend: «Man verfuhr zu strenge mit ihm. Sanft ruhe seine Asche. Lange wird sein Name noch genennet werden, wenn die, so über ihn herfielen, längst vergessen sind.» Denn aus der Sicht dieses Fürsprechers werden «wenige Werke wol so allgemeinen und verdienten Beyfall erhalten, als dieses [von der Erfahrung]. Es ist ein klassisches Buch geworden [...] Ich bitte jeden Arzt, dies Werk [von der Einsamkeit] zu lesen. Jeder Arzt muss es lesen, er wird viel daraus lernen ${ }^{61}$.

Die Ähnlichkeit in den ärztlichen Auffassungen zwischen einem Arzt der Aufklärungszeit und Ärzten, die eine vitalistische Medizin vertraten, zeigt, dass es wesentlich engere Verflechtungen zwischen der Medizin der Aufklärung und der des Vitalismus gab, als nachfolgende Generationen angenommen haben.

$59 \mathrm{Vgl}$. Anm. 12.

60 Aus einem Brief vom 17. Oktober 1757: «Ich schreibe von der Erfahrung in den Arzneiwissenschaften und ich als Verächter der Logik schreibe, so dass ich auf der einen Seite den Bakon und Locke auf der anderen den Hippokrates und Sydenham liegen habe.» Ischer a.a.O. (Anm. 4), S. 267.

61 Imanuel Vertraugott Rothe: Handbuch für die medizinische Litteratur nach allen ihren Theilen oder Anleitung zur Kenntnis der besten auserlesenen medicinischen Bücher. Leipzig 1799, S. 348. 